\title{
The Fate of Unruptured Intracranial Vertebrobasilar Dissecting Aneurysm with Brain Stem Compression According to Different Treatment Modalities
}

\author{
(DD.Y. Cho, (D)B.-S. Kim, (D).H. Choi, (D)Y.K. Park, and (D)Y.S. Shin
}

\begin{abstract}
BACKGROUND AND PURPOSE: Unruptured intracranial vertebrobasilar dissecting aneurysms with brain stem compression are difficult to treat. In the present study, the clinical and radiologic outcomes of unruptured intracranial vertebrobasilar dissecting aneurysms with brain stem compression based on different treatment modalities were evaluated.
\end{abstract}

MATERIALS AND METHODS: This study included 28 patients with unruptured intracranial vertebrobasilar dissecting aneurysms with brain stem compression treated from January 2009 to December 2017. Treatment methods were observation $(n=6)$, stent-assisted coil embolization $(n=9)$, parent artery occlusion $(n=6)$, and flow diversion $(n=7)$. The data of baseline characteristics, change of aneurysm size, retreatment rate, stroke occurrence, and alteration of the mRS score were obtained from retrospective chart review.

RESULTS: The initial size of dissecting aneurysms was largest in the flow diversion group (22.5 $\pm 7.7 \mathrm{~mm})$, followed by parent artery occlusion $(20.3 \pm 8.4 \mathrm{~mm})$, stent-assisted coil embolization $(11.7 \pm 2.2 \mathrm{~mm})$, and observation $(17.8 \pm 5.5 \mathrm{~mm} ; P=.01)$ groups. The reduction rate of aneurysm size was highest in the parent artery occlusion group $(26.7 \pm 32.1 \%)$, followed by flow diversion $(14.1 \% \pm 28.7 \%)$, stent-assisted coil embolization $(-17.9 \pm 30.3 \%)$, and observation $(-31.5 \pm 30.8 \% ; P=.007)$ groups. Additional treatment was needed in the observation $(4 / 6,66.7 \%)$ and stent-assisted coil embolization $(3 / 9,33.3 \% ; P=.017)$ groups. Improvement of the mRS score on follow-up was observed in the flow diversion (6/7, 85.7\%) and parent artery occlusion (4/6, 66.7\%) groups but not in the stent-assisted coil embolization and observation groups. A worsened mRS score was most common in the observation group $(4 / 6,66.7 \%)$, followed by stent-assisted coil embolization $(3 / 9,33.3 \%)$, parent artery occlusion $(2 / 6,33.3 \%)$, and flow diversion $(0 / 7,0 \%)$ groups.

CONCLUSIONS: When treating intracranial vertebrobasilar dissecting aneurysms with brain stem compression, parent artery occlusion and flow diversion should be considered to reduce aneurysm size and improve the mRS score.

ABBREVIATIONS: FD = flow diversion; IVBDA = intracranial vertebrobasilar dissecting aneurysm; PAO = parent artery occlusion; SACE $=$ stent-assisted coil embolization

ntracranial vertebrobasilar dissecting aneurysm (IVBDA) is one of the most common intracranial arterial dissections. On initiation of a sudden disruption of the internal elastic lamina and media, circulating blood invades the disrupted layer, resulting in formation of an intramural hematoma. ${ }^{1}$ When the

Received May 17, 2019; accepted after revision August 20.

From the Department of Neurosurgery (D.Y.C.), Ewha Womans University Seoul Hospital, College of Medicine, Ewha Womans University, Seoul, Republic of Korea; Departments of Radiology (B.-S.K.) and Neurosurgery (J.H.C., Y.S.S.), Seoul St.

Mary's Hospital, College of Medicine, The Catholic University of Korea, Seoul, Republic of Korea; and Department of Neurosurgery (Y.K.P.), Ilsan Paik Hospital, College of Medicine, Inje University, Goyang, Republic of Korea.

Please address correspondence to Yong Sam Shin, MD, PhD, Department of Neurosurgery, Seoul St. Mary's Hospital, The Catholic University of Korea, 222 Banpo-daero, Seocho-gu, Seoul 137-701, Republic of Korea; e-mail: nsshin@gmail.com

http://dx.doi.org/10.3174/ajnr.A6252 intramural hematoma extends toward the outer layer, the dissection presents as aneurysmal dilation due to hemodynamic stress to separated adventitia from invading circulating blood. ${ }^{2}$ The aneurysmal dilation of intracranial arterial dissection may progress to subarachnoid hemorrhage with adventitial tearing. Moreover, a large IVBDA may induce brain stem compression due to the position adjacent to the brain stem, which might result in a neurologic deficit. Thus, treatment for unruptured large IVBDAs with brain stem compression should target the prevention of aneurysm rupture and relief of mass effect on the brain stem. Various treatment modalities, such as conservative, endovascular, and surgical treatments are available for IVBDAs. Possible surgical treatment options for IVBDA are reconstruction with direct clipping or proximal ligation; however, when the aneurysm is large, these options are commonly limited due to 
Table 1: Baseline characteristics of patients in the 4 different treatment technique groups $^{\mathrm{a}}$

\begin{tabular}{lccccc}
\multicolumn{1}{c}{ Variables } & $\begin{array}{c}\text { Observation } \\
(\boldsymbol{n}=6)\end{array}$ & $\begin{array}{c}\text { SACE } \\
(\boldsymbol{n}=\mathbf{9})\end{array}$ & $\begin{array}{c}\text { PAO } \\
(\boldsymbol{n}=6)\end{array}$ & $\begin{array}{c}\text { FD } \\
(\boldsymbol{n}=\mathbf{7})\end{array}$ & $\begin{array}{c}\boldsymbol{P} \\
\text { Value }\end{array}$ \\
\hline Age (yr) & $58.2 \pm 8.3$ & $54.3 \pm 14.7$ & $45.3 \pm 15.7$ & $47.6 \pm 22.1$ & $.47^{\mathrm{b}}$ \\
Sex, male & $3(50)$ & $3(33.3)$ & $2(33.3)$ & $2(28.6)$ & $.87^{\mathrm{c}}$ \\
DM & $0(0)$ & $1(11.1)$ & $0(0)$ & $0(0)$ & $.51^{\mathrm{c}}$ \\
Hypertension & $3(50)$ & $4(44.4)$ & $3(50)$ & $1(14.3)$ & $.47^{\mathrm{c}}$ \\
Dyslipidemia & $2(33.3)$ & $0(0)$ & $0(0)$ & $2(28.6)$ & $.14^{\mathrm{c}}$ \\
$\quad \begin{array}{l}\text { Current smoker } \\
\text { Symptoms }\end{array}$ & $1(16.7)$ & $0(0)$ & $0(0)$ & $2(28.6)$ & $.23^{\mathrm{c}}$ \\
$\quad$ Incidental & $1(16.7)$ & $5(55.6)$ & $0(0)$ & $0(0)$ & $.04^{\mathrm{c}}$ \\
$\quad$ Headache & $3(50)$ & $2(22.2)$ & $2(33.3)$ & $1(14.3)$ & \\
$\quad$ Neurologic deficit & $2(33.3)$ & $2(22.2)$ & $4(66.7)$ & $6(85.7)$ & \\
Location & $2(33.3)$ & $4(44.4)$ & $1(16.7)$ & $2(28.6)$ & \\
$\quad$ Right VA & $2(33.3)$ & $5(55.6)$ & $3(50)$ & $3(42.9)$ & \\
$\quad$ Left VA & $2(33.3)$ & $0(0)$ & $2(33.3)$ & $2(28.6)$ & \\
$\quad$ BA & & & & & \\
\hline
\end{tabular}

Note:-DM indicates diabetes mellitus; VA, vertebral artery; BA, basilar artery.

${ }^{a}$ Values are presented as mean \pm SD or number (\%).

${ }^{\mathrm{b}}$ ANOVA.

${ }^{\mathrm{c}}$ Fisher exact test.

difficult access and postoperative complications. Because of these surgical limitations, diverse endovascular treatments for large vertebrobasilar dissecting aneurysms are currently performed, such as stent-assisted coil embolization (SACE), parent artery occlusion (PAO), and, recently, flow diversion (FD). ${ }^{3-6}$ In the present study, clinical and radiologic outcomes of unruptured IVBDAs with brain stem compression based on different treatment modalities were evaluated.

\section{MATERIALS AND METHODS}

All patient data from a single institution (Seoul St. Mary's Hospital) were retrospectively reviewed, and data collection was approved by the institutional review board.

\section{Patients}

From January 2009 to December 2017, two hundred seventy-two IVBDAs were diagnosed in a single institution. Large IVBDAs with brain stem compression were diagnosed on the basis of the following criteria: 1) angiographic evidence of IVBDA (ie, aneurysmal dilation or a pearl-and-string sign of the vertebrobasilar artery on $3 \mathrm{D}$-reconstruction digital subtraction angiography); 2) radiologic evidence of IVBDA on MR imaging (ie, intramural hematoma, double lumen sign, intimal flap); and 3) mass effect of an aneurysm compressing the brain stem, with distortion of boundary shape on MR imaging. Exclusion criteria were the following: 1) a ruptured IVBDA; 2) traumatic IVBDA; 3) iatrogenic IVBDA; 4) laboratory or radiologic findings suggestive of vasculopathy, fibromuscular dysplasia, or Bechet disease; and 5) patients lost to follow-up. All clinical and radiologic data, including age, sex distribution, risk factors (diabetes mellitus, hypertension, dyslipidemia, current smoker), location of the IVBDA, and follow-up period were collected retrospectively.

\section{Treatment Modalities}

Patients included in this study underwent 4 different treatment modalities: 1) observation, 2) SACE, 3) PAO, or 4) FD. Patients who refused endovascular treatment due to procedure-related complications underwent observation and were closely followed up in the outpatient clinic. These patients were counseled regarding a $30 \%$ risk of mortality and morbidity based on pre-

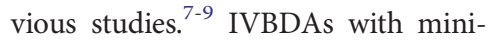
mal brain stem compression due to the relatively small size were typically treated with SACE. PAO and FD were performed when the IVBDA was large and the lesion was symptomatic. PAO was considered first when the branching arteries were not incorporated and enough collateral flow was expected after occlusion of the parent artery. FD was considered when postoperative ischemia was expected after PAO due to poor collateral flow and branching artery involvement. All endovascular treatments were performed with the patient under general anesthesia with right femoral artery puncture; aspirin $(100 \mathrm{mg}$ ) and clopidogrel (75 mg) were given daily for 7 days before treatment. Intravenous heparin was administered during the procedure.

An Enterprise 2 self-expanding stent (Codman \& Shurtleff, Raynham, Massachusetts) or a LVIS Blue or Jr (MicroVention, Tustin, California) with detachable coils was used for SACE, and PAO was performed with detachable coils. All SACE cases were treated with a conventional stent-assisted coiling using a single stent. The Pipeline Embolization Device (PED; Covidien, Irvine, California) or the Flow-Redirection Endoluminal Device (FRED; MicroVention) was used for FD. In the endovascular treatment groups, dual-antiplatelet medication with $100 \mathrm{mg}$ of aspirin and $75 \mathrm{mg}$ of clopidogrel was maintained for at least 6 months and was gradually reduced in the outpatient clinic. After 6 months of dual-antiplatelet medication, the SACE and PAO groups maintained a single antiplatelet regimen with $100 \mathrm{mg}$ of aspirin for 18 more months, and the FD group maintained a single antiplatelet regimen with $100 \mathrm{mg}$ of aspirin life-long.

\section{Radiologic and Clinical Follow-Up}

The patients in SACE, PAO, and FD groups underwent diffusion-weighted MR imaging on postoperative day 1 for evaluation of postprocedural infarction. Follow-up MR imaging was performed in all patients after 6, 12, and 24 months to evaluate changes in aneurysm size. Additional MR imaging was performed at 2-year intervals or if new neurologic symptoms developed. The preprocedural and postprocedural sizes of aneurysms were measured on axial sections of T2 sequence MR imaging, which presents the largest IVBDA diameter. The size of the IVBDA on the initial and last MR imaging examination was recorded, and the percentage of size reduction was calculated. Associated infarction on the initial clinical diagnosis of IVBDA and occurrence of newly developed infarctions during the followup period were recorded. The mRS score on the initial and last follow-up was recorded, and improvement or deterioration of the mRS was analyzed. 
Table 2: Radiologic and clinical outcomes of patients in the 4 different treatment technique groups ${ }^{\mathrm{a}}$

\begin{tabular}{|c|c|c|c|c|c|}
\hline Variables & $\begin{array}{c}\text { Observation } \\
(n=6)\end{array}$ & $\begin{array}{l}\text { SACE } \\
(n=9)\end{array}$ & $\begin{array}{l}\text { PAO } \\
(n=6)\end{array}$ & $\begin{array}{c}\text { FD } \\
(n=7)\end{array}$ & $\begin{array}{c}P \\
\text { Value }\end{array}$ \\
\hline Follow-up (mo) & $28.3 \pm 14.2$ & $27 \pm 23.4$ & $40.2 \pm 37.4$ & $16.3 \pm 13.1$ & $.37^{\mathrm{b}}$ \\
\hline Initial aneurysm size (mm) & $17.8 \pm 5.5$ & $11.7 \pm 2.2$ & $20.3 \pm 8.4$ & $22.5 \pm 7.7$ & $.01^{\mathrm{b}}$ \\
\hline Aneurysm size on follow-up (mm) & $23.4 \pm 9.8$ & $13.9 \pm 5.2$ & $14.4 \pm 9.7$ & $19.9 \pm 10.1$ & $.1^{\mathrm{b}}$ \\
\hline Change of aneurysm size (\%) & $129.3 \pm 29.5$ & $117.8 \pm 30.0$ & $73.2 \pm 32.2$ & $85.9 \pm 28.7$ & $.008^{\mathrm{b}}$ \\
\hline Retreatment & $4(66.7)$ & $3(33.3)$ & $0(0)$ & $0(0)$ & $.017^{\mathrm{c}}$ \\
\hline SACE & $0(0)$ & $1(33.3)$ & $0(0)$ & $0(0)$ & \\
\hline PAO & $1(25)$ & $0(0)$ & $0(0)$ & $0(0)$ & \\
\hline FD & $3(75)$ & $2(66.6)$ & $0(0)$ & $0(0)$ & \\
\hline Initial ischemic stroke & $0(0)$ & 1 (11.1) & $1(16.7)$ & $1(14.3)$ & $.79^{\mathrm{c}}$ \\
\hline Ischemic stroke on follow-up & $1(16.7)$ & $1(11.1)$ & $1(16.7)$ & $0(0)$ & $.73^{\mathrm{c}}$ \\
\hline Subarachnoid hemorrhage on follow-up & $0(0)$ & $0(0)$ & $0(0)$ & $0(0)$ & \\
\hline Improvement of mRS score & $0(0)$ & $0(0)$ & $4(66.7)$ & $6(85.7)$ & $<.001^{\mathrm{c}}$ \\
\hline Worsening of mRS score & $4(66.7)$ & $3(33.3)$ & $2(33.3)$ & $0(0)$ & $.086^{\mathrm{c}}$ \\
\hline
\end{tabular}

${ }^{a}$ Values are presented as mean \pm SD or number (\%).

${ }^{\mathrm{b}}$ ANOVA test.

${ }^{\mathrm{C}}$ Fisher exact test.

\section{Statistical Analysis}

Statistical analysis was performed using SPSS, Version 24 (IBM, Armonk, New York). Baseline characteristics and radiologic and clinical outcomes between different treatment technique groups were analyzed. One-way ANOVA with post hoc Bonferroni corrections and the Kruskal-Wallis test were used for numeric variables, and the Fisher exact test was used for nominal variables. $P$ values $<.05$ were considered statistically significant.

\section{RESULTS}

\section{Baseline Characteristics of Patients in the 4 Different Treatment Technique Groups}

Among the 272 IVBDAs, 40 IVBDAs met the inclusion criteria. Twelve IVBDAs were excluded due to ruptured lesions $(n=6)$, history of trauma $(n=1)$, suggestive vasculopathy $(n=1)$, and loss to follow-up $(n=4)$. Finally, 28 patients with IVABADs were identified and enrolled in the study. Among the 28 patients with IVBDAs with brain stem compression, 6 patients underwent observation, 9 patients underwent SACE, 6 patients underwent $\mathrm{PAO}$, and 7 patients underwent FD. Table 1 shows baseline characteristics of patients in each treatment technique group. Significant differences in age, male sex distribution, risk factors, and follow-up period were not observed. None of the basilar artery lesions were treated with SACE, and no significant difference was observed in the location of IVBDAs. Initial presenting symptoms were significantly different between the groups. The proportion of IVBDAs without symptoms was highest in the SACE group, and all IVBDAs treated with PAO and FD were symptomatic. The proportion of headache as an initial symptom was highest in the observation group, and the proportion of neurologic deficits was highest in the FD group $(P=.04)$.

\section{Radiologic and Clinical Outcomes of Patients in the 4 Different Treatment Technique Groups}

The mean age of patients was $51.5 \pm 16$ years (range, 12-71 years), and the mean follow-up period was $27.4 \pm 23.8$ months. Table 2 shows radiologic and clinical outcomes of patients in the 4 different treatment-technique groups. The initial IVBDA size was significantly different between the groups. The initial size

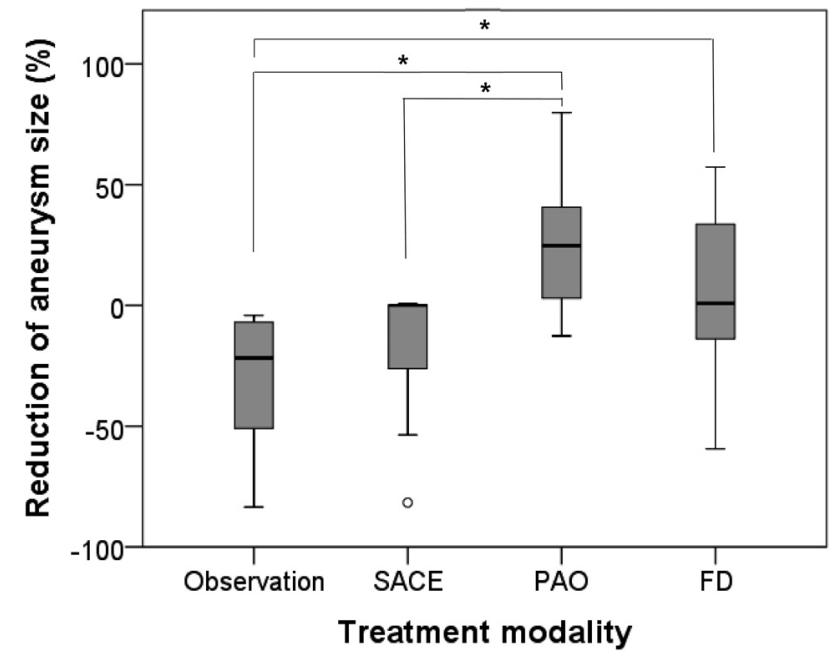

FIG 1. Reduction of vertebrobasilar dissecting aneurysm size based on treatment technique. The PAO group showed the greatest amount of aneurysm reduction followed by FD, SACE, and observation groups. The asterisk indicates $P<.05$.

was largest in the FD group $(22.5 \pm 7.7 \mathrm{~mm})$, followed by PAO $(20.3 \pm 8.4 \mathrm{~mm})$, observation $(17.8 \pm 5.5 \mathrm{~mm})$, and SACE $(11.7 \pm 2.2 \mathrm{~mm} ; P<.01)$ groups. The change of IVBDA size was significantly different among the groups $(P=.008)$. The reduction of IVBDA size on follow-up compared with initial size was observed in the PAO $(73.2 \% \pm 32.2 \%)$ and FD $(85.9 \pm 28.7 \%)$ groups. Conversely, increased IVBDA size was observed in the SACE $(117.8 \% \pm 30 \%)$ and observation $(129.3 \% \pm 29.5 \%)$ groups. The comparison of the reduction rate of aneurysm size is shown in Fig 1. The PAO group showed the highest rate of size reduction $(73.2 \% \pm 32.2 \%)$, followed by $\mathrm{FD}(14.1 \% \pm 28.7 \%)$, SACE $(-17.9 \% \pm 30.3 \%)$, and observation $(-31.5 \% \pm 30.8 \%)$ groups. The PAO group showed a significantly large rate of size reduction compared with the SACE $(P=.047)$ and observation $(P=.014)$ groups. The size-reduction rate in the FD group was significantly different compared with the observation group $(P=.05)$.

The outlier in the SACE group in Fig 1 is shown in Fig 2. A 20 -year-old man presented with headache, and MR imaging and 

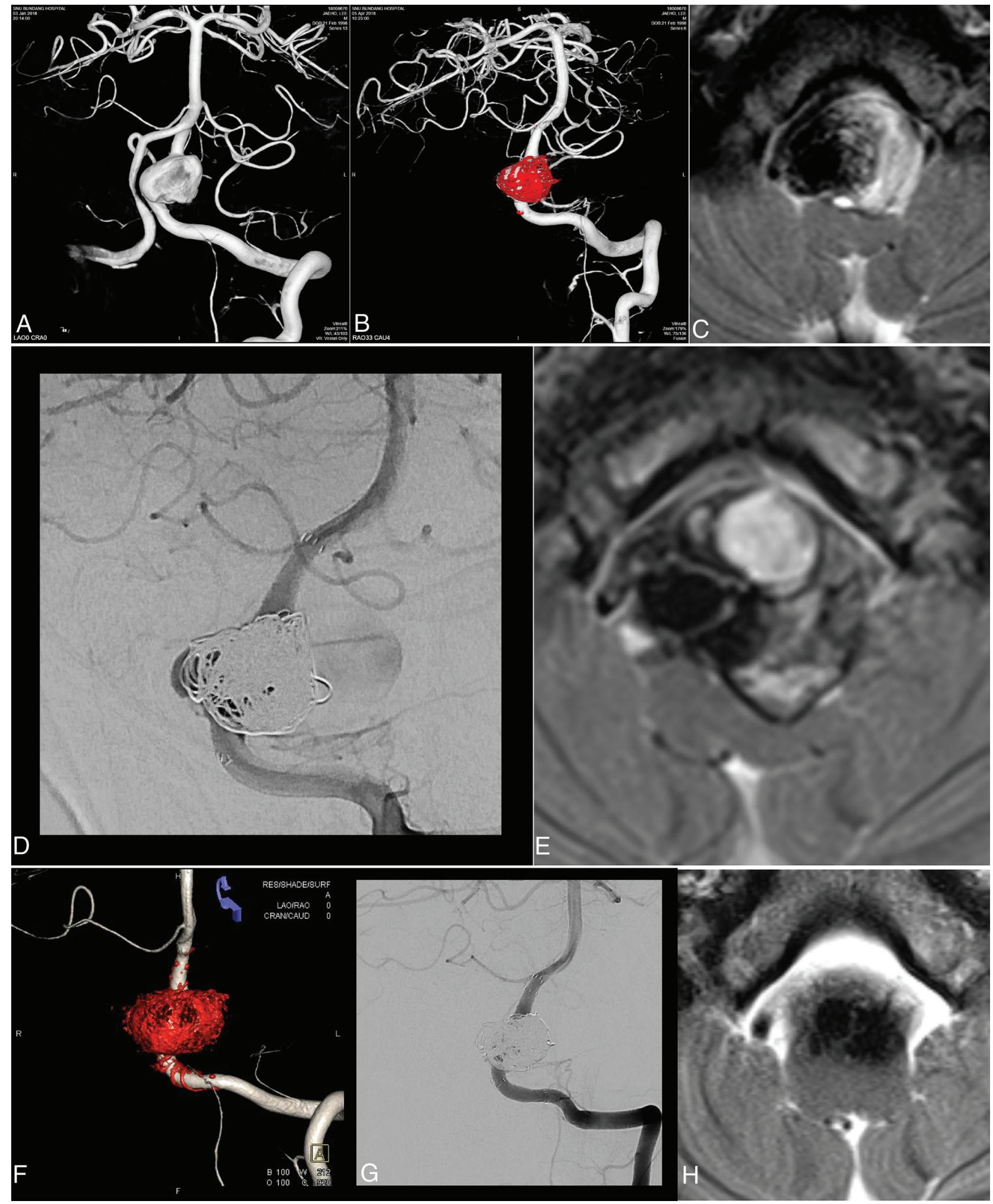

FIG 2. Treatment of large vertebral artery dissecting aneurysm with brain stem compression in a 20-year-old man. A, 3D rotational angiography shows a large dissecting aneurysm on the fourth segment of the right vertebral artery. B, Stent-assisted coil embolization was performed. C, T2 axial MR image on postoperative day 12 shows coil meshes with extended thrombus in the aneurysm sac. $D$, Angiography after 3 months shows a jet flow into aneurysm sac. $E$, T2 axial MR image shows enlargement of aneurysm diameter with aggravation of brain stem compression. $F$, FD was performed using the FRED. G, Angiography 5 months after FD shows complete regression of jet inflow. $H$, T2 axial MR imaging shows a markedly decreased aneurysm sac diameter with diminished brain stem compression. 


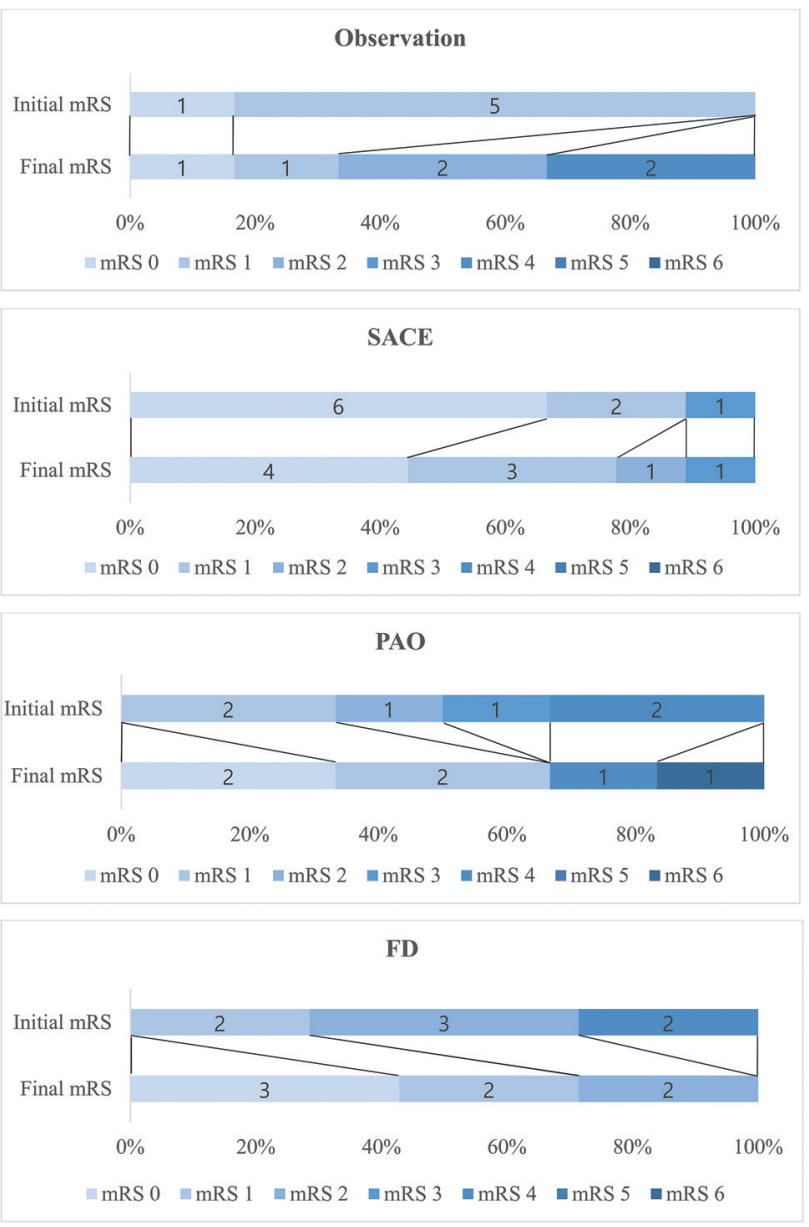

FIG 3. Alteration of mRS based on the treatment technique.

angiography showed a fusiform dissecting aneurysm on the left V4. SACE was initially performed but resulted in an $82 \%$ increase of aneurysm size after 3 months with development of a neurologic deficit. The patient was retreated with FD with excellent outcome. Among the 28 IVBDAs with brain stem compression, 4 of $6(66.7 \%)$ observation patients and 3 of 9 (33.3\%) patients treated with SACE underwent retreatment due to aggravation of brain stem mass effect and a worsened mRS score. Conversely, no patient needed additional treatment in the PAO and FD groups. These values were statistically significant $(P=.017)$. Among the 4 retreated patients in the observation group, 1 underwent PAO and 3 underwent FD. Among the 3 patients treated with SACE, 1 underwent additional coil embolization and 2 underwent FD. Patients additionally treated with PAO or FD showed good outcomes with cessation of aneurysm growth; however, the aggravation of mass effect due to aneurysm growth was evident in patients who underwent additional coil embolization.

Among the 28 IVBDAs with brain stem compression, 3 associated ischemic strokes were observed on initial diagnosis: 1 transient ischemic attack in the SACE $(1 / 9,11.1 \%)$ and PAO $(1 / 6$, $16.7 \%)$ groups and 1 cerebellar infarction in the FD (1/7, 14.3\%) group. On follow-up, there were 3 infarctions: 1 cerebellar infarction in the observation group $(1 / 6,16.7 \%), 1$ medullary infarction in the SACE group on postoperative day $7(1 / 9,11.1 \%)$, and 1 cerebellar infarction in the PAO group on postoperative day 1 $(1 / 6,16.7 \%)$; no ischemic stroke occurred in the FD group. Initial and follow-up stroke rates were not statistically different among treatment modalities $(p=0.79, p=0.73)$.

Ten patients showed improved mRS scores. The improvement rate of mRS scores was highest in the FD group $(6 / 7,85.7 \%)$ followed by the PAO group $(4 / 6,66.7 \%)$. The observation and SACE groups showed no mRS improvement. These values were statistically significant $(P<.001)$. Lower mRS scores were observed in 9 patients. The rate of worsened mRS scores was highest in the observation group $(4 / 6,66.7 \%)$, followed by the SACE $(3 / 9,33.3 \%)$ and PAO $(2 / 6,33.3 \%)$ groups. A worsened $\mathrm{mRS}$ score was not observed in the FD group. However, the difference between the worsened rates of mRS scores was not statistically significant $(P=.086)$. The changes in mRS scores based on treatment technique is shown in Fig 3. mRS scores in the observation and SACE groups showed a tendency to improve, whereas the mRS scores in the PAO and FD groups tended to decrease. Among the 28 patients, 1 patient in the PAO group died. The 47-year-old man with hypertension and end-stage renal disease was diagnosed with a large thrombosed dissecting aneurysm on the basilar artery. The patient was treated with PAO; however, the aneurysm continued to grow, and the patient progressed to a vegetative state due to brain stem compression and obstructive hydrocephalus after 5 months. The patient died after 8 months due to complications from pneumonia and sepsis caused by multiorgan failure.

\section{DISCUSSION}

Large IVBDAs typically have a poor prognosis due to the high risk of rupture and growth characteristics. Still, there is a question about the treatment technique as to whether such patients should undergo reconstructive or destructive treatment. A systematic review and meta-analysis showed higher angiographic occlusion rates for a destructive technique and lower morbidity rates for a reconstruction technique, but long-term neurologic outcomes and retreatment rates were not statistically different. ${ }^{10}$ In the present study, we compared clinical and radiologic outcomes of various destructive and reconstructive treatments and compared them with each other and with an observation group.

The baseline characteristics of 4 different treatment-technique groups did not show a significant difference except for initial symptoms and the initial size of the IVBDAs. In our treatment strategy, IVBDAs with relatively small sizes and no symptoms were mostly treated with SACE to prevent ischemic complications and obtain immediate aneurysm occlusion. Large IVBDAs inducing a neurologic deficit were mostly treated with $\mathrm{PAO}$ or FD to avoid a mass effect caused by the coil mesh and to decrease the aneurysm size to relieve the neurologic deficit caused by brain stem compression. The initial size of IVBDAs in the observation group was higher than that in the SACE group. This finding might be due to patients who refused to undergo treatment or untreatable lesions due to large size with prominent brain stem perforators and incorporation of cerebellar arteries before approval of the FD device. 


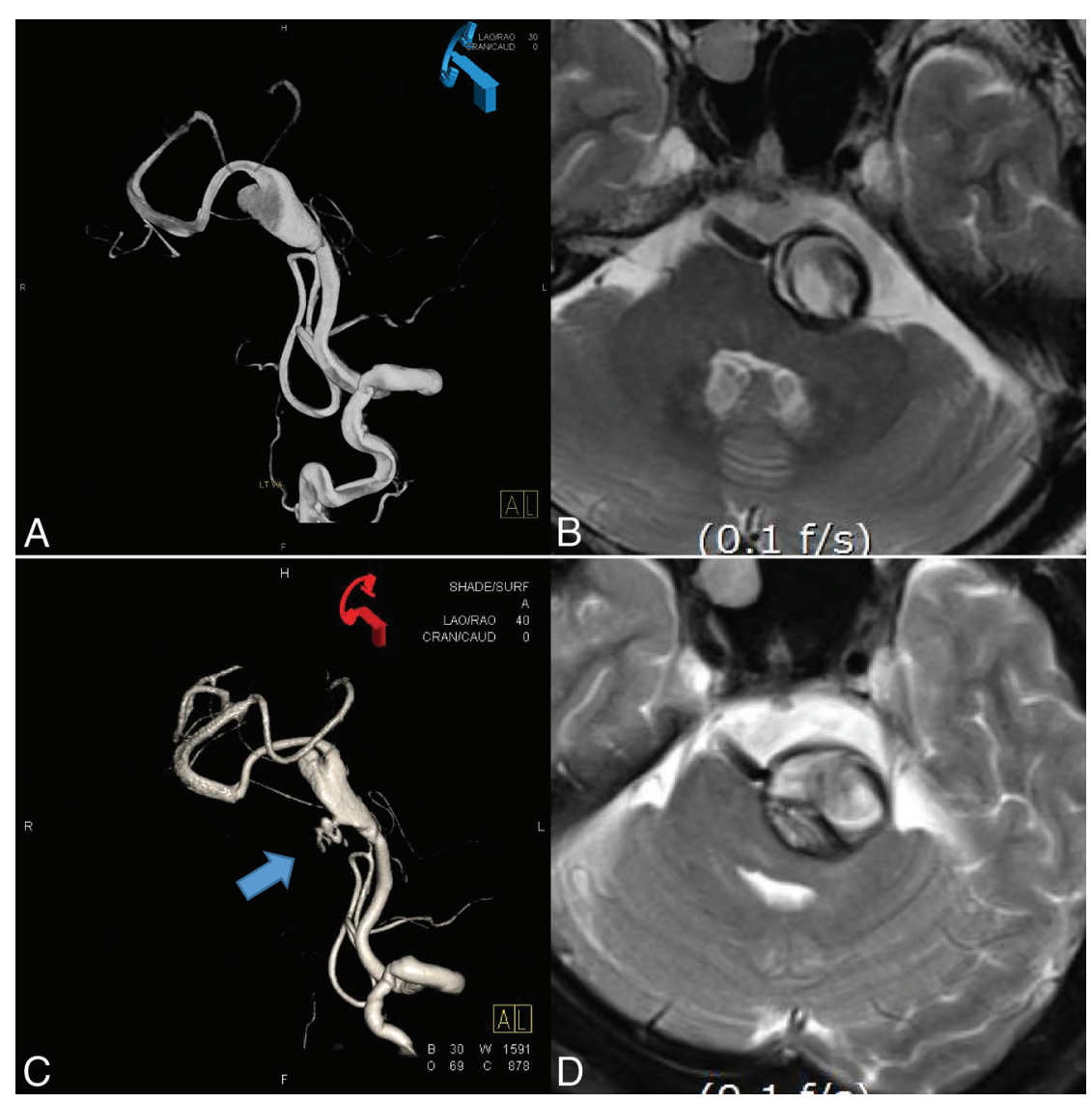

FIG 4. Growth of an observed vertebral artery dissecting aneurysm in a 67-year-old woman. A, $3 \mathrm{D}$ rotational angiography shows a fusiform-type vertebral artery dissecting aneurysm on the fourth segment of left vertebral artery. $B, T 2$ axial MR image shows formation of intra-aneurysmal thrombus with brain stem compression. The patient underwent observation, and right-sided weakness developed after 5 years. C, 3D rotational angiography after 5 years shows contrast extension into the thrombosed aneurysm sac (arrow). D, T2 axial MR image shows enlargement of aneurysm diameter with aggravation of brain stem compression.

The natural course of vertebrobasilar dissection is benign. ${ }^{2,11,12}$ The nature of an unruptured IVBDA is also not highly aggressive. Kai et $\mathrm{al}^{13}$ reported that only 9 of 100 unruptured IVBDAs needed surgical or endovascular treatment due to progression of symptoms or change of angiographic shape during 24 months of follow-up. However, the prognosis of large IVBDAs is worse than that of stenotic-type dissections or small IVBDAs. Kobayashi et $\mathrm{al}^{14}$ reported the need for treatment of IVBDAs larger than $10 \mathrm{~mm}$ due to aneurysm growth and clinical deterioration caused by a mass effect. Our study showed similar results, with the largest size increase and highest rate of mRS deterioration in the observation group. In addition to aggravation of a mass effect to the brain stem in growing IVBDAs, the high risk of rupture should also be considered. Naito et $\mathrm{al}^{15}$ reported a higher-than-expected risk of bleeding in large or growing vertebral artery dissections (3/21), and Flemming et $\mathrm{al}^{16}$ reported enlargement of a dissecting aneurysm as a risk factor of rupture. In our study, 4 of 6 patients in the observation group eventually underwent endovascular treatment due to aneurysm enlargement and clinical deterioration caused by a mass effect to the brain stem. One of the progressed cases of a large IVBDA with brain stem compression is described in Fig 4.
Surgical treatment of IVBDAs with brain stem compression was a unique option before approval of endovascular devices. Drake and Peerless ${ }^{17}$ reported outcomes of 120 giant fusiform intracranial aneurysms with surgical treatment: Fiftysix were located in basilar trunk, vertebrobasilar junction, and vertebral artery. They were treated with surgical clipping, wrapping, or proximal ligation with or without bypass surgery. Four patients showed severe neurologic deficits, and 13 patients died. Due to the high mortality and morbidity of surgical treatment, endovascular treatments have emerged as treatments of choice. In our study, all IVBDAs with brain stem compression were treated endovascularly.

Although endovascular treatment of the IVBDAs shows lower mortality and morbidity rates compared with surgical treatment, various perioperative complications can occur. Destructive treatment might induce perioperative ischemic stroke due to occlusion of perforators or branching arteries. Reconstructive treatment also might cause ischemic and hemorrhagic stroke. SACE has the possibility of perioperative aneurysm rupture during aneurysm selection or coil deployment and thromboembolic infarction due to the deployment of stent. FD might cause delayed rupture of aneurysms and perforator infarction. The recent meta-analysis comparing the destructive technique with the reconstructive technique in treating IVBDAs showed similar perioperative mortality and morbidity. ${ }^{10}$ In our study, the ischemic stroke rate during follow-up was higher in the deconstructive group $(1 / 6,16.7 \%)$ than in the reconstructive group (1/16, $6.3 \%)$, but it was not statistically significant due to small variables. No perioperative hemorrhagic stroke occurred in any treatment modality.

Stent-assisted coil embolization has been widely performed to treat vertebrobasilar dissecting aneurysms because of immediate aneurysm occlusion with preservation of parent artery flow. Jeon et $\mathrm{al}^{18}$ reported the feasibility and safety of SACE in IVBDAs, with acceptable long-term follow-up results despite a high rate of incomplete immediate occlusion. In addition, 25 of 47 lesions (53.2\%) showed no immediate contrast filling; during a mean follow-up of 20.2 months, 37 of 47 lesions (78.7\%) appeared completely occluded. However, a high rate of immediate incomplete occlusion can be problematic in treating large IVBDAs with SACE. Because most large IVBDAs have a wide neck, it is difficult to achieve complete packing of the aneurysm, leading to coil 


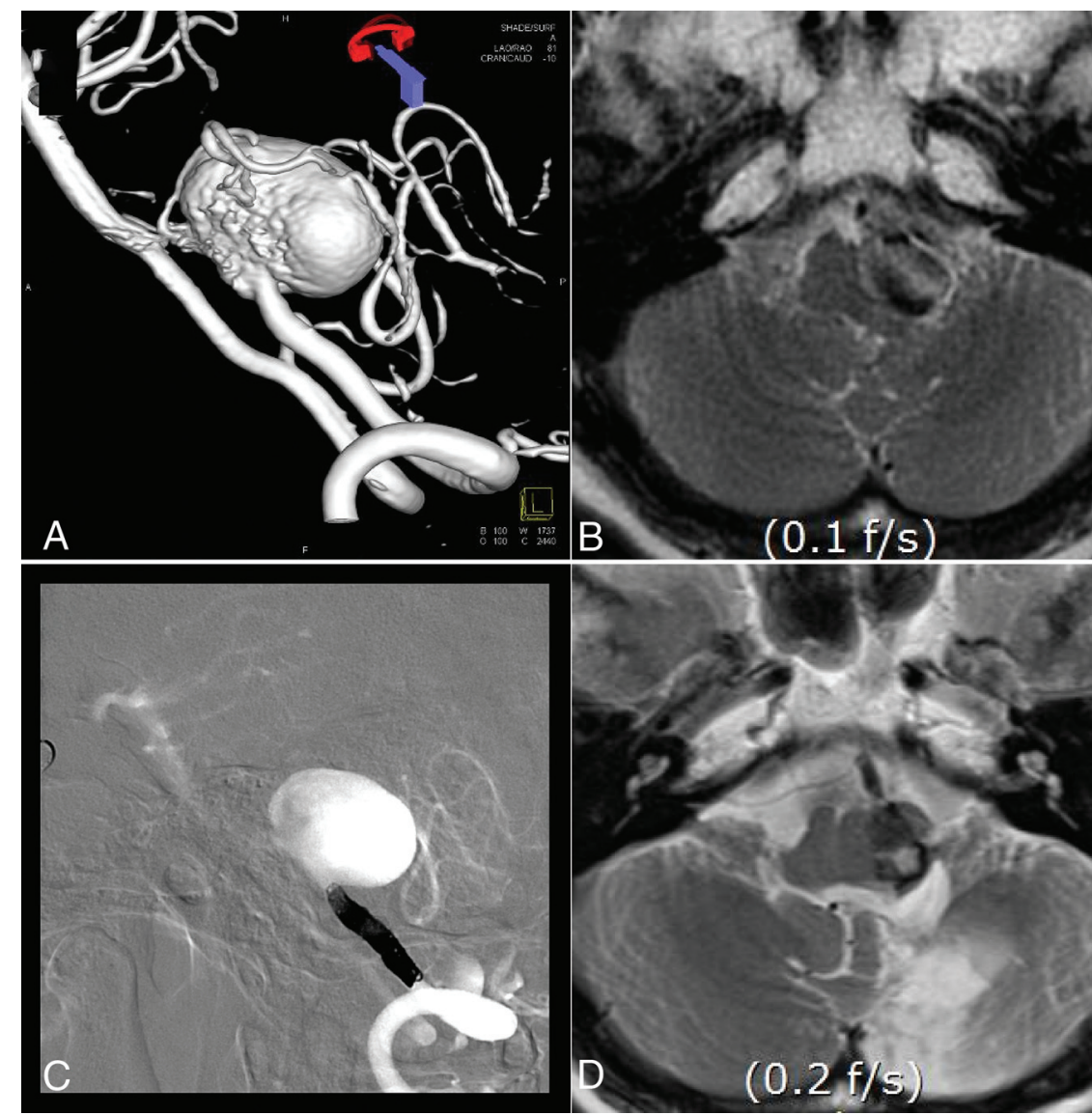

FIG 5. Reduction of vertebral artery dissecting aneurysm size using PAO in 58-year-old woman. $A$, Rotational $3 D$ angiography shows a large vertebral artery dissecting aneurysm in the fourth segment of the left vertebral artery. $B, T 2$ axial MR image shows thrombus formation with brain stem compression. C, PAO was performed. D, T2 axial MR imaging after 12 months shows a decreased diameter of the aneurysm with diminished brain stem compression.

thrombosed holobasilar aneurysms. The rest of the 4 patients in the PAO group showed mRS improvement. The risk of postoperative infarction in the PAO group was not significantly higher than in the other treatment groups. The mean reduction of aneurysm size was $73.2 \%$, which is the largest among the treatment groups. One of the cases with reduction of IVBDA size using parent artery occlusion is described in Fig 5.

The efficacy of FD for anterior circulation aneurysms has been demonstrated in numerous studies, ${ }^{23-25}$ but pessimism remains regarding FD for posterior circulation aneurysms because of concern for perforator infarction and delayed rupture. Siddiqui et al reported unfavorable outcome in 7 patients with symptomatic large or giant fusiform vertebrobasilar aneurysms treated with flow diverters. Of these, 4 patients died (2 with delayed rupture and 2 with brain stem infarction), and 1 patient had an $\mathrm{mRS}$ score of 5. Preoperative stroke and multiple uses of an FD device were risk factors for postoperative perforator infarction. ${ }^{26}$ On the other hand, there are some reports presenting favorable outcomes. Natarajan et $\mathrm{al}^{27}$ reported that 11 of 12 fusiform vertebrobasilar aneurysms treated with FD compaction and reopening of the aneurysm. Furthermore, intraaneurysmal thrombus is common in large IVBDAs and results in coil migration and loosening into the thrombosed portion. ${ }^{19,20}$ These failures result in growth of the IVBDA with aggravating brain stem compression. In our study, the initial mean aneurysm size of the SACE group was $11.7 \mathrm{~mm}$, which increased to $13.9 \mathrm{~mm}$ on follow-up. Three of 9 patients $(33.3 \%)$ treated with SACE showed mRS deterioration or no mRS improvement.

Parent artery occlusion might be a good treatment option in large IVBDAs with brain stem compression if branching arteries are not incorporated. There are numerous studies of PAO in the treatment of IVBDAs. Aymard et $\mathrm{al}^{21}$ reported a good outcome of PAO in unclippable vertebrobasilar aneurysms, and 13 of 21 IVBDAs showed good clinical and angiographic outcomes. Leibowitz et $\mathrm{al}^{22}$ reported a high rate of aneurysm occlusion with PAO when complete thrombosis of the aneurysm was achieved. IVBDAs involving bilateral vertebral arteries or the basilar artery showed poor prognosis compared with unilateral vertebral artery involvement due to difficulties in thrombus formation caused by abundant collateral flow. In our study, 2 of 6 patients (33.3\%) in the PAO group showed mRS deterioration: 1 of 4 patients with a unilateral vertebral artery lesion (25\%) and 1 of 2 patients with a basilar artery lesion (50\%). Both basilar aneurysms were showed recovery of neurologic deficits, and all showed aneurysm occlusion at the final follow-up. This study suggests the importance of patient selection because they did not include patients with holobasilar aneurysms. We treated 2 basilar trunk aneurysms with flow diversion, both of which were midbasilar, not holobasilar aneurysms. Both patients with basilar aneurysms treated with FD showed improvement of the mRS and aneurysm size reduction. One case showing recurrent IVBDA after SACE is presented in Fig 2, and the other was a 10-year-old girl without intra-aneurysmal thrombus and with initial brain stem infarction. We achieved total occlusion with a single FD device without ischemic stroke on follow-up in both cases.

The limitations of this study are that it was retrospective and performed in a single center with a small number of patients with discordant initial variables, which may lead to statistical bias. Second, the prognoses of vertebral artery dissecting aneurysm and basilar artery aneurysm were not separately analyzed, despite their different disease natures. Third, there is lack of evidence to prove the superiority of PAO or FD in treating IVBDAs with brain stem compression. The rate of aneurysm size reduction was higher in the PAO group, and the proportion of patients with $\mathrm{mRS}$ improvement was higher in the FD group. The PAO group showed 2 of 6 with mRS worsening, and the FD group did not 
show any mRS worsening. However, these differences between the 2 groups were not statistically significant due to a small number of variables. Moreover, FD sometimes causes unexpected postoperative aneurysm rupture due to a change of hemodynamics, which is fatal. Fortunately, our FD group did not show any postoperative delayed rupture of aneurysm, but the risk remains. Further study with a larger population is needed.

\section{CONCLUSIONS}

Unruptured IVBDAs with brain stem compression aggravate neurologic deficits and increase in size when untreated, indicating the need for active treatment. The SACE technique does not prevent aneurysm growth or neurologic deterioration in many cases. Thus, PAO and FD should be considered to obtain consistent reduction of aneurysm size and improvement of mRS.

\section{REFERENCE}

1. Park KW, Park JS, Hwang SC, et al. Vertebral artery dissection: natural history, clinical features and therapeutic considerations. $J$ Korean Neurosurg Soc 2008;44:109-15 CrossRef Medline

2. Mizutani T. Natural course of intracranial arterial dissections. $J$ Neurosurg 2011;114:1037-44 CrossRef Medline

3. Jin SC, Kwon DH, Choi CG, et al. Endovascular strategies for vertebrobasilar dissecting aneurysms. AJNR Am J Neuroradiol 2009; 30:1518-23 CrossRef Medline

4. Guerrero WR, Ortega-Gutierrez S, Hayakawa M, et al. Endovascular treatment of ruptured vertebrobasilar dissecting aneurysms using flow diversion embolization devices: single-institution experience. World Neurosurg 2018;109:e164-69 CrossRef Medline

5. Leibowitz R, Do HM, Marcellus ML, et al. Parent vessel occlusion for vertebrobasilar fusiform and dissecting aneurysms. AJNR Am J Neuroradiol 2003;24:902-07 Medline

6. Lylyk P, Ceratto R, Hurvitz D, et al. Treatment of a vertebral dissecting aneurysm with stents and coils: technical case report. Neurosurgery 1998;43:385-88 CrossRef Medline

7. Mu S, Li C, Yang X, et al. Reconstructive endovascular treatment of spontaneous symptomatic large or giant vertebrobasilar dissecting aneurysms: clinical and angiographic outcomes. Clin Neuroradiol 2016;26:291-300 CrossRef Medline

8. Steinberg GK, Drake CG, Peerless SJ. Deliberate basilar or vertebral artery occlusion in the treatment of intracranial aneurysms: immediate results and long-term outcome in 201 patients. J Neurosurg 1993;79:161-73 CrossRef Medline

9. van Oel LI, van Rooij WJ, Sluzewski M, et al. Reconstructive endovascular treatment of fusiform and dissecting basilar trunk aneurysms with flow diverters, stents, and coils. AJNR Am J Neuroradiol 2013;34:589-95 CrossRef Medline

10. Sönmez Ö, Brinjikji W, Murad MH, et al. Deconstructive and reconstructive techniques in treatment of vertebrobasilar dissecting aneurysms: a systematic review and meta-analysis. AJNR Am J Neuroradiol 2015;36:1293-98 CrossRef Medline
11. Kitanaka C, Tanaka J, Kuwahara M, et al. Nonsurgical treatment of unruptured intracranial vertebral artery dissection with serial follow-up angiography. J Neurosurg 1994;80:667-74 CrossRef Medline

12. Nakagawa K, Touho $H$, Morisako $T$, et al. Long-term follow-up study of unruptured vertebral artery dissection: clinical outcomes and serial angiographic findings. J Neurosurg 2000;93:1925 CrossRef Medline

13. Kai Y, Nishi T, Watanabe M, et al. Strategy for treating unruptured vertebral artery dissecting aneurysms. Neurosurgery 2011;69:108591; discussion 1091-92 CrossRef Medline

14. Kobayashi N, Murayama Y, Yuki I, et al. Natural course of dissecting vertebrobasilar artery aneurysms without stroke. AJNR Am J Neuroradiol 2014;35:1371-75 CrossRef Medline

15. Naito I, Iwai T, Sasaki T. Management of intracranial vertebral artery dissections initially presenting without subarachnoid hemorrhage. Neurosurgery 2002;51:930-37; discussion 937-38 CrossRef Medline

16. Flemming KD, Wiebers DO, Brown RD Jr, et al. Prospective risk of hemorrhage in patients with vertebrobasilar nonsaccular intracranial aneurysm. J Neurosurg 2004;101:82-87 CrossRef Medline

17. Drake CG, Peerless SJ. Giant fusiform intracranial aneurysms: review of 120 patients treated surgically from 1965 to $1992 . J$ Neurosurg 1997;87:141-62 CrossRef Medline

18. Jeon JP, Cho YD, Rhim JK, et al. Stent-assisted coil embolization of vertebrobasilar dissecting aneurysms: procedural outcomes and factors for recanalization. Korean J Radiol 2016;17:801-10 CrossRef Medline

19. van Rooij WJ, Sluzewski M. Endovascular treatment of large and giant aneurysms. AJNR Am J Neuroradiol 2009;30:12-18 CrossRef Medline

20. Sluzewski M, Menovsky T, van Rooij WJ, et al. Coiling of very large or giant cerebral aneurysms: long-term clinical and serial angiographic results. AJNR Am J Neuroradiol 2003;24:257-62 CrossRef Medline

21. Aymard A, Gobin YP, Hodes JE, et al. Endovascular occlusion of vertebral arteries in the treatment of unclippable vertebrobasilar aneurysms. J Neurosurg 1991;74:393-98 CrossRef Medline

22. Leibowitz R, Do HM, Marcellus ML, et al. Parent vessel occlusion for vertebrobasilar fusiform and dissecting aneurysms. AJNR Am J Neuroradiol 2003;24:902-07 Medline

23. Fischer S, Vajda Z, Aguilar Perez M, et al. Pipeline embolization device (PED) for neurovascular reconstruction: initial experience in the treatment of 101 intracranial aneurysms and dissections. Neuroradiology 2012;54:369-82 CrossRef Medline

24. Lylyk P, Miranda C, Ceratto R, et al. Curative endovascular reconstruction of cerebral aneurysms with the Pipeline embolization device: the Buenos Aires experience. Neurosurgery 2009;64:632-43 CrossRef Medline

25. Nelson P, Lylyk P, Szikora I, et al. The Pipeline embolization device for the intracranial treatment of aneurysms trial. AJNR Am J Neuroradiol 2011;32:34-40 CrossRef Medline

26. Siddiqui AH, Abla AA, Kan P, et al. Panacea or problem: flow diverters in the treatment of symptomatic large or giant fusiform vertebrobasilar aneurysms. J Neurosurg 2012;116:1258 CrossRef Medline

27. Natarajan SK, Lin N, Sonig A, et al. The safety of Pipeline flow diversion in fusiform vertebrobasilar aneurysms: a consecutive case series with longer-term follow-up from a single US center. $J$ Neurosurg 2016;125:111-19 CrossRef Medline 